\title{
MicroRNAs and Hematopoietic Differentiation
}

\author{
A. Fatica, ${ }^{*}$ A. Rosa, ${ }^{*}$ F. Fazi ${ }^{\dagger}$ M. Ballarino, ${ }^{*}$ M. Morlando, ${ }^{*}$ F.G. De Angelis, ${ }^{*}$ \\ E. Caffarelli, ${ }^{*}$ C. Nervi, ${ }^{\dagger}$ and I. Bozzoni* \\ *Institute Pasteur Cenci-Bolognetti, Department of Genetics and Molecular Biology and I.B.P.M., University of \\ Rome "La Sapienza," 00185 Rome, Italy; 'Department of Histology and Medical Embryology, University of Rome \\ "La Sapienza" and San Raffaele Bio-medical Science Park of Rome, 00128 Rome, Italy
}

\begin{abstract}
The discovery of microRNAs (miRNAs) and of their mechanism of action has provided some very new clues on how gene expression is regulated. These studies established new concepts on how posttranscriptional control can fine-tune gene expression during differentiation and allowed the identification of new regulatory circuitries as well as factors involved therein. Because of the wealth of information available about the transcriptional and cellular networks involved in hematopoietic differentiation, the hematopoietic system is ideal for studying cell lineage specification. An interesting interplay between miRNAs and lineage-specific transcriptional factors has been found, and this can help us to understand how terminal differentiation is accomplished.
\end{abstract}

The completion of the human genome sequencing together with the development of new genome-wide approaches has provided very powerful tools for identifying gene products whose expression could be correlated with cell growth and differentiation. Nevertheless, these studies have concentrated on expression profiles of mRNAs or proteins. In the last few years, several new classes of small noncoding RNAs have been identified, and quite a lot of data have been accumulated on their functions in regulating gene expression at the posttranscriptional level, including alternative splicing, mRNA stability, and translation. These events are very relevant in cell metabolism, growth, and differentiation not only because they provide a means for expanding protein diversity and abundance, but also because they enable the cell to rapidly respond to external stimuli and to adjust the biosynthetic machineries to the different growth requirements. Inside the small noncoding RNA family, particular interest is now devoted to miRNAs. These molecules are synthesized by endogenous cellular genes and have been shown to regulate mRNA and protein abundance by controlling both the stability and the translation of the target mRNAs (Pillai et al. 2005). Since the characterization of the first miRNA (Lee et al. 1993), several hundred different miRNAs have been identified, and many data are now available on their role in regulatory circuits controlling developmental timing, cell death, cell proliferation, apoptosis, hematopoiesis, and patterning of the nervous system (Ambros 2004). The existence of numerous tissue- and developmentalstage-specific miRNAs and the evolutionary conservation of many miRNAs argue for numerous additional, yet unidentified, functions of this class of transcripts. Notably, miRNA activity has also been correlated with cancer since miRNAs with oncogenic and tumor suppressor activity have been identified and a new molecular taxonomy of human cancers based on miRNA profiling has been proposed (Caldas and Brenton 2005).
Hundreds of miRNAs operating in different organisms have been identified through cloning and genetics, and through bioinformatic methods. According to current predictions, 800 or more miRNAs operate in primates, and each miRNA may target dozens of mRNAs. Hence, it is estimated that expression of as many as $30 \%$ of human genes may be controlled by miRNAs (Lewis et al. 2005).

miRNA-coding regions have very peculiar and heterogeneous genomic organizations. Many of them are found in intronic regions and may be transcribed as part of the host gene; however, the majority are located in intergenic regions or in annotated genes but in an antisense orientation, strongly suggesting that they form independent transcription units. miRNAs have been shown to be transcribed by RNA polymerase II (RNA pol II) from transcriptional units that differ from those of protein-coding genes in that they do not possess canonical TATA boxes and are intronless. Nowadays, very little is known about their transcriptional regulation, including the factors responsible for basal and tissue-specific expression.

Some miRNAs show ubiquitous expression (Sempere et al. 2004), but others are limited to certain stages in development or to certain tissues and cell types (Lee and Ambros 2001). The study of tissue-specific miRNAs will therefore provide important clues for identifying new sets of genes and regulatory circuits involved in the control of cell-specific differentiation.

Hematopoiesis is a lifelong, highly regulated multistage process where a pluripotent self-renewing hematopoietic stem cell (HSC) gives rise to all blood cell lineages. In addition, the hematopoietic system is ideal for identifying miRNAs and regulatory factors involved in cell lineage specification, since many transcriptional and cellular networks have been already identified as crucial for differentiation. We describe here examples of how miRNAs can influence cell lineage specification and how the identification of their target mRNAs has contributed to the understanding of the molecular networks involved in the alternative control between cell growth and differentiation. 
Ectopic expression and knockdown of specific miRNAs have provided powerful molecular tools able to control the switch between proliferation and differentiation, thereby also providing new potential therapeutic tools for interfering with tumorigenesis.

\section{IDENTIFICATION OF MIRNAS SPECIFICALLY EXPRESSED DURING HEMATOPOIESIS AND LEUKEMIA}

Expression profiling analysis showed that most miRNAs are under the control of developmental or tissuespecific signaling. Transcriptional regulation is likely to be the major control step of miRNA expression, even if some miRNAs seem to be controlled at the posttranscriptional level (Bartel 2004; Kim 2005; Gregory and Shiekhattar 2005).

Despite their small size, miRNA expression is analyzed by standard techniques for RNA expression studies, including northern blot and quantitative polymerase chain reaction (PCR). To analyze the relative expression level of miRNAs from different tissues, large-scale cDNA cloning has been successfully utilized, even if for high-throughput analysis the most widely used method is based on microarrays (Croce and Calin 2005). However, it should be mentioned that microarray is not as quantitative as northern blot so that in most of the cases, it is not easy to exactly define the relative abundance of individual miRNAs.

In past years, several specific miRNAs differentially expressed during hematopoietic differentiation have been identified and shown to have an important role in mammalian hematopoiesis. The first case was described in mice: miR-181, miR-223, and miR-142 are differentially expressed in hematopoietic tissues, and their expression is regulated during hematopoiesis and lineage commitment (Chen et al. 2004). Notably, ectopic expression of miR-181 in murine hematopoietic progenitor cells led to an increased fraction of B-lymphoid cells in both tissue culture differentiation assays and adult mice, suggesting a central role of miRNAs in the control of hematopoietic lineage differentiation.

Since then, many miRNA profiles have been derived by either microarray or conventional northern analysis on different hematopoietic lineages. One of the most complete miRNA expression profiles was recently obtained by a new bead-based flow cytometric method (Lu et al. 2005). In this study, the miRNA expression detected in normal tissue was globally higher than that in tumors, corroborating the idea that the miRNA expression profile reflects the differentiation state of the cell. miRNA expression was also profiled during differentiation of primary hematopoietic progenitor cells and myeloid cell lines into erythrocytes and granulocytes, respectively. As predicted, blood cell differentiation was always associated with global changes in miRNA expression (Lu et al. 2005). Analysis on specific members of the miRNA family allowed their role to be distinguished either in cell proliferation or in differentiation. In humans, miR-221 and miR222 have been shown to be down-modulated in human $\mathrm{CD}_{3} 4^{+}$cord blood progenitor cells induced toward the erythroid lineage. During early stages of erythroid differentiation that are coupled with exponential growth, Kit protein levels are high, although they decrease in terminal erythroblasts undergoing little proliferation. The expression levels of both miR-221 and miR-222 are inversely related to the amount of the Kit protein, strongly suggesting its posttranscriptional regulation during expansion of early erythroblasts (see Fig. 1). Functional studies indeed indicated that the decline of miR-221 and miR-222 unblocks Kit protein production at the translational level, leading to expansion of early erythroid precursors. The decrease of Kit protein at later differentiation stages, where little proliferation occurs, is instead due to transcriptional down-regulation (Felli et al. 2005). In agreement with these findings, treatment of $\mathrm{CD} 34^{+}$progenitors with miR221 and miR-222, via oligonucleotide transfection or lentiviral vector infection, caused impaired proliferation

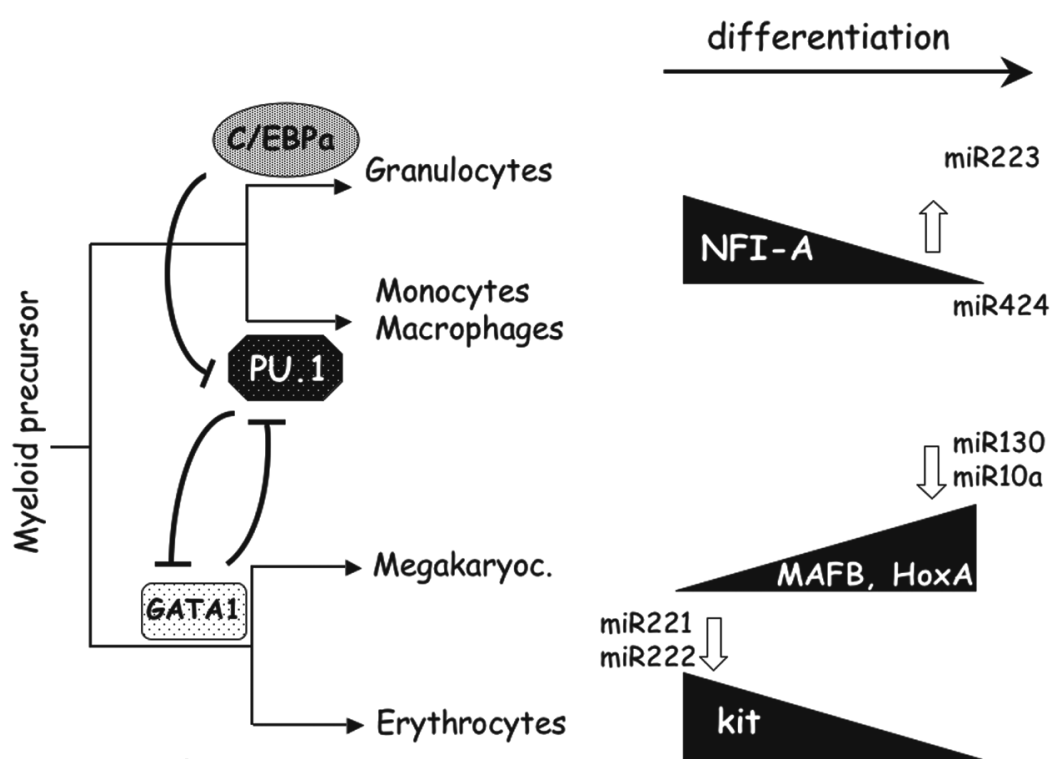

Figure 1. The transcriptional factors responsible for the commitment to the four myeloid terminal differentiation lineages are indicated. These proteins have been shown to inhibit each other's function by physical interaction. The miRNAs that undergo specific up- and down- (open arrows) regulation are indicated in correspondence with the effect exerted on their respective validated target. In the case of the Kit protein, its decrease at later differentiation stages is due to transcriptional down-regulation. 
and accelerated differentiation of erythropoietic cultures, coupled with down-modulation of the Kit protein (Felli et al. 2005), suggesting an important role for both miRNAs in controlling the proliferation and differentiation status of early erythroblasts.

Down-regulation of miR-130 correlated instead with the up-regulation of the MAFB transcriptional factor during megakaryocytic differentiation, and the decrease of miR-10 paralleled the increase of the HOXA1 factor (Garzon et al. 2006). Interestingly, the synthesis of both MAFB and HoxA1 has been correlated with terminal commitment of megakaryocytic cells. Compared with the previous class, miR-223 was shown to increase upon granulocytic differentiation and to promote maturation of promyelocytic precursors: In fact, its ectopic expression in APL cells induced commitment to the granulocytespecific lineage, whereas its depletion counteracted differentiation (Fazi et al. 2005). miR-223 activation correlated in time with the decrease in accumulation of the NFI-A protein while its mRNA levels remained unaltered; this, together with the experimental validation of the bona fide target site in the 3'UTR (untranslated region), indicated that NFI-A is a target of miR-223. Interestingly, the NFI-A factor was previously implicated in replication as well as in controlling changes in cell growth (Gronostajski 2000), and its knockdown was shown to enhance granulocytic differentiation (Fazi et al. 2005). NFI-A mRNA is also a target of other miRNAs up-regulated during monocyte/macrophage differentiation, such as miR-424 (Kasashima et al. 2004), therefore indicating that its repression is very important for at least two myeloid differentiation pathways.

Figure 1 summarizes a few examples of regulatory networks that correlate up- and down-regulation of miRNA expression relative to their targets during different lineages of myeloid differentiation. From the few cases studied so far, it can be suggested that those miRNAs decreasing during lineage commitment unblock the expression of key proteins required for differentiation, whereas those increasing during maturation have a negative effect on growth-promoting factors. Along this line, several lines of evidence link miRNAs to leukemias. The human miR-15a/miR-16 cluster is frequently deleted or down-regulated in patients with B-cell chronic lymphocytic leukemia (Calin et al. 2005). These miRNAs negatively regulate BCL2, which is an antiapoptotic gene that is often overexpressed in many types of human leukemias. Another miRNA, miR-155, is up-regulated in many pediatric and adult lymphomas, especially in diffuse large B-cell lymphomas, Hodgkin lymphomas, and certain types of Burkitt lymphomas (Metzler et al. 2004; Eis et al. 2005). Recently, it has been shown that miR-155 transgenic mice exhibit initially a preleukemic pre-B-cell proliferation evident in spleen and bone marrow, followed by frank B-cell malignancy. These findings indicate that the role of miR-155 is to induce polyclonal expansion, favoring the capture of secondary genetic changes for full transformation (Costinean et al. 2006).

Another example is provided by an aggressive B-cell leukemia in which Myc is translocated downstream from
miR-142, disrupting the miRNA synthesis and increasing Myc expression by the miRNA promoter. It is now clear that miRNAs may function as oncogenes and tumor suppressors (Hammond 2006); therefore, studies into miRNA expression and function might lead to an advanced understanding of the mechanisms involved in tumorigenesis.

\section{ACTIVATORS OF MIRNA EXPRESSION IN HEMATOPOIESIS}

Several transcriptional factors have been shown to have a crucial role in hematopoiesis. Important information has been obtained from overexpression and knockout experiments. Some of these factors, such as SCL/Tal1 and AML1, are common to all lineages, and their depletion affects the entire blood cell lineage. Among the factors that have lineage-specific expression patterns are GATA1, the CAAT/enhancer-binding protein $\mathrm{C} / \mathrm{EBP} \alpha$, and PU.1. GATA1 was the first "lineage-specific" factor studied in detail and shown to be essential for erythroid and megakaryocytic lineages. PU.1 mediates lymphoid and monocyte/macrophage differentiation, and $\mathrm{C} / \mathrm{EBP} \alpha$ has a more specific function in granulopoiesis (Radomska et al. 1998). Interestingly, these factors have some functional interconnection since $\mathrm{C} / \mathrm{EBP} \alpha$ and GATA1 can inhibit PU.1 function (Radomska et al. 1998; Reddy et al. 2002), whereas increases in PU.1 lead to inhibition of GATA1 (Fig. 1).

A variety of studies in different experimental systems have provided compelling evidence indicating a very important role of $\mathrm{C} / \mathrm{EBP} \alpha$ in both growth arrest and terminal differentiation, in particular of granulocytes (Nerlov et al. 1998; Radomska et al. 1998; Wang et al. 1999; Khanna-Gupta et al. 2001; Nakajima and Ihle 2001). Although $\mathrm{C} / \mathrm{EBP} \alpha$ was known to induce granulopoiesis while suppressing monocyte differentiation, it was unclear how $\mathrm{C} / \mathrm{EBP} \alpha$ regulated this cell fate choice at the mechanistic level. In addition, mice deficient for known $\mathrm{C} / \mathrm{EBP} \alpha$ target genes did not exhibit the same block in granulocyte maturation, indicating the necessity of identifying additional $\mathrm{C} / \mathrm{EBP} \alpha$ target genes essential for myeloid cell development.

In this view, it was very interesting to find that the miR-223 promoter contained two binding sites for the $\mathrm{C} / \mathrm{EBP} \alpha$ factor and to show that this region was essential for the up-regulation of the $\mathrm{miR}$ in response to retinoic acid treatment. ChIP (chromatin immunoprecipitation) experiments have indeed also shown that the binding of this factor, whose synthesis and binding ability are induced upon retinoic acid treatment, correlated with differentiation and the up-regulation of miR-223 expression (Fazi et al. 2005).

A search in the miR-223 promoter also revealed the presence of an NFI-A site (Meisterernst et al. 1988; Bachurski et al. 1997) overlapping one of the two $\mathrm{C} / \mathrm{EBP} \alpha$-binding sites. ChIP experiments demonstrated that this factor is indeed binding the miR-223 promoter but only in the absence of retinoic acid. Therefore, $\mathrm{C} / \mathrm{EBP} \alpha$ and NFI-A factors compete for the same site, 

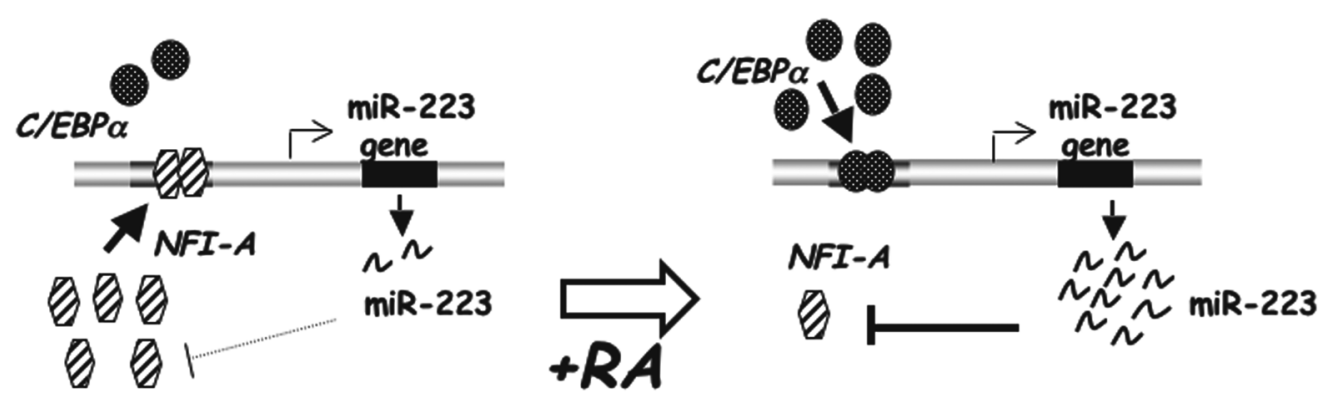

Figure 2. Schematic model of the autoregulatory loop controlling miR-223 expression. $(A)$ In promyelocytic cells, NFI-A keeps low levels of miR-223 expression and, consequently, low miR-223-mediated translational repression. (B) Upon retinoic acid treatment, $\mathrm{C} / \mathrm{EBP} \alpha$ replaces NFI-A on the miR-223 promoter and up-regulates miR-223. This results in (1) an increase of miR-223-mediated translational repression of the NFI-A mRNA, (2) a decrease of the NF1-A protein, and (3) stimulation of granulocytic differentiation.

and their binding correlates with low (NFI-A) and high transcriptional activity of miR-223. Since NFI-A is also the target of miR-223, a very interesting autoregulatory loop appears to control granulocytic differentiation: $\mathrm{C} / \mathrm{EBP} \alpha$ activation produces the displacement of NFI-A from the miR-223 promoter and its up-regulation. In cascade, miR-223 acts by repressing NFI-A, thus subtracting it from the competition with $\mathrm{C} / \mathrm{EBP} \alpha$ and maintaining sustained levels of miR-223 expression (Fig. 2).

RNAi against either factor not only validated their activity with respect to miR-223 transcription, but also indicated their different roles in differentiation. NFI-A is required during proliferation of the precursor cells and counteracts differentiation, whereas $\mathrm{C} / \mathrm{EBP} \alpha$ is required for differentiation into granulocytes. These findings are in agreement with the roles described for $\mathrm{C} / \mathrm{EBP} \alpha$ and NFI-A. C/EBP $\alpha$ has been implicated in promoting differentiation and repressing genes that have the important function in stimulating growth (McKnight 2001; Tenen 2001); on the other side, NFI-A has been implicated in replication as well as in controlling changes in cell growth (Gronostajski 2000).

In analogy with the role of $\mathrm{C} / \mathrm{EBP} \alpha$ in activating the expression of lineage-specific miRNAs, it was interesting to discover that several miRNAs specifically expressed during monocyte/macrophage differentiation (Kasashima et al. 2004) contained PU.1-binding sites in their promoter. Even more striking was the fact that some of these miRNAs have among their targets the NFI-A mRNA. These findings are very interesting in view of the fact that PU.1 has been described as a major player in the commitment to this differentiation lineage and of the important role of NFI-A in repressing differentiation. Therefore, it can be concluded that in both granulocyte and monocyte/macrophage differentiation, lineage-specific transcriptional factors activate miRNAs that inhibit the expression of NFI-A, thus allowing differentiation to proceed.

\section{NFI-A FACTOR}

NFI-A was initially described as a CCAAT-boxbinding transcription factor, belonging to the nuclear factor I (NFI) family of proteins (Santoro et al. 1988). The NFI family is composed of four independent genes
(NFI-A, -B, -C, and -X) and a large number of splice variants that form homodimers and heterodimers, thus creating an extensive network of possible functional dimers (Gronostajski 2000). The NFI proteins bind as dimers to the dyad symmetric consensus sequence TTGGC(N5)GCCAA; nevertheless, they could also bind very well to individual half-sites even with a reduced affinity and presumably as dimers with other proteins (Meisterernst et al. 1988; Bachurski et al. 1997).

Figure 3B shows the results of RT-PCR performed to identify the different NFI-A mRNA species expressed in HeLa cells and in the APL cell line NB4. Both cell lines display two types of NFI-A transcripts, one corresponding to the full length and the other to an isoform lacking exon 10 (black box). Skipping this exon results in changing of the reading frame, thus producing a protein variant in the carboxy-terminal portion. So far, since trans-activation/repression activities have been assigned to this domain, it is not known whether the two variants differ in such properties. Interestingly, both forms can undergo posttranscriptional control since they have the same 3'UTR with several target sequences for several classes of miRNAs. Figure 3A indicates that according to miRanda algorithm (John et al. 2004), five miRNA families are able to recognize the $3^{\prime}$ UTR of NFI-A. Among them, miR-223 is specifically expressed during granulocytic differentiation, where it has been demonstrated to indeed repress NFI-A (Fazi et al. 2005). The miR-424 family is instead specifically activated in the monocyte/macrophage pathway; interestingly, the activation of miR-424 correlates with the decrease of NFI-A levels during monocyte differentiation (Fig. 3C), suggesting that it has a direct role in the down-regulation of this protein. From these observations, it can be suggested that if NFI-A repression is a general theme for differentiation, this is accomplished in the different hematopoietic lineages through activation of specific classes of miRNAs.

In conclusion, the identification of hematopoietic lineagespecific miRNAs has allowed us to unravel regulatory circuitries and factors therein involved that altogether form the complex network of interactions responsible for the commitment and maintenance of the different hematopoietic programs. The study of the regulation of these networks will also enable the identification of crucial 


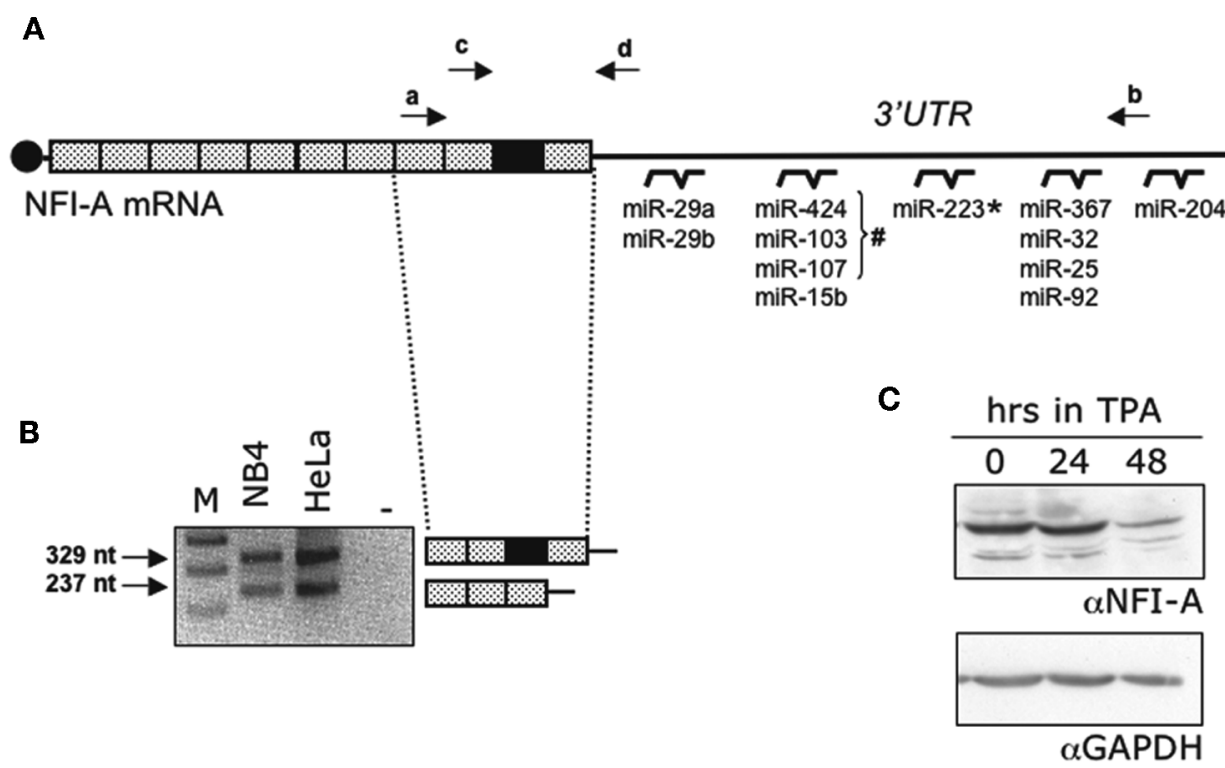

Figure 3. (A) Schematic representation of the NFI-A mRNA. (Black box) Alternatively spliced exon 10. miRNA target sites in the 3'UTR are shown. (\#) miRNAs induced by TPA; $(*)$ miRNAs induced by retinoic acid. $(B)$ Nested RT-PCR on poly $(A)^{+}$RNA extracted from HeLa and NB4 cell lines. An oligo(dT) was used in the retrotranscription reaction; the resulting cDNA was initially amplified with primers annealing on exon 8 and the $3^{\prime}$ UTR (primers a and b, respectively, in panel $A$ ); $2 \mu 1$ of a 1:100 dilution of this amplification was used for a second PCR with primers specific for exon 9 and for an inner region of the $3^{\prime}$ UTR (primers c and d, respectively, in panel A). The amplification products from HeLa and NB4 cell lines were run on a 1.2\% agarose gel in parallel with a negative contol (lane). The 329-nucleotide-long amplification product corresponds to the full-length transcript, whereas that of 237 nucleotides corresponds to the transcript lacking exon 10. A schematic representation of the two products is shown on the side of the gel. (Lane M) Molecularweight marker. $(C)$ NB4 cells were treated with $16 \mathrm{nM}$ TPA for the indicated times; $50 \mu \mathrm{g}$ of protein was analyzed by western blot with anti-NFI-A antibody (upper panel) and anti-GAPDH antibody as a loading control (lower panel).

factors whose modulation can be utilized to control aberrant proliferation as in the case of several leukemias.

\section{ACKNOWLEDGMENTS}

This work was partially supported by grants from AIRC and AIRC-ROC, Sixth Research Framework Programme of the European Union, Project RIGHT (LSHB-CT-2004 005276), MURST (FIRB-p.n. RBNE015MPB and RBNE01KXC9 to I.B.), PRIN, and "Centro di eccellenza BEMM."

\section{REFERENCES}

Ambros V. 2004. The function of animal miRNAs. Nature 431: 350 .

Bachurski C.J., Kelly S.E., Glasser S.W., and Currier T.A. 1997. Nuclear factor I family members regulate the transcription of surfactant protein-C. J. Biol. Chem. 272: 32759.

Bartel D.P. 2004. MicroRNAs: Genomics, biogenesis, mechanism, and function. Cell 116: 281.

Caldas C. and Brenton J.D. 2005. Sizing up miRNAs as cancer genes. Nat. Med. 11: 712.

Calin G.A., Ferracin M., Cimmino A., Di Leva G., Shimizu M., Wojcik S.E., Iorio M.V., Visone R., Sever N.I., Fabbri M., et al. 2005. A microRNA signature associated with prognosis and progression in chronic lymphocytic leukemia. N. Engl. J. Med. 353: 1793.

Chen C.Z., Li L., Lodish H.F., and Bartel D.P. 2004. MicroRNAs modulate hematopoietic lineage differentiation. Science 303: 83 .

Costinean S., Zanesi N., Pekarsky Y., Tili E., Volinia S., Heerema N., and Croce C.M. 2006. Pre-B cell proliferation and lymphoblastic leukemia/high-grade lymphoma in E $\mu$ miR155 transgenic mice. Proc. Natl. Acad. Sci. 103: 7024.

Croce C.M. and Calin G.A. 2005. miRNAs, cancer, and stem cell division. Cell 122: 6 .

Eis P.S., Tam W., Sun L., Chadburn A., Li Z., Gomez M.F., Lund E., and Dahlberg J.E. 2005. Accumulation of miR-155 and BIC RNA in human B cell lymphomas. Proc. Natl. Acad. Sci. 102: 3627.

Fazi F., Rosa A., Fatica A., Gelmetti V., De Marchis M.L., Nervi C., and Bozzoni I. 2005. A minicircuitry comprised of microRNA-223 and transcription factors NFI-A and C/EBPalpha regulates human granulopoiesis. Cell 123: 819 .

Felli N., Fontana L., Pelosi E., Botta R., Bonci D., Fachiano F., Liuzzi F., Lulli V., Morsili O., Santoro S., et al. 2005. MicroRNAs 221 and 222 inhibit normal erythropoiesis and erythroleukemic cell growth via kit receptor downmodulation. Proc. Natl. Acad. Sci. 102: 18081.

Garzon R., Pichiorri F., Palumbo T., Iuliano R., Cimmino A., Aqeilan R., Volinia S., Bhatt D., Alder H., Marcucci G., et al. 2006. MicroRNA fingerprints during human megakaryocytopoiesis. Proc. Natl. Acad. Sci. 103: 5078.

Gregory R.I. and Shiekhattar R. 2005. MicroRNA biogenesis and cancer. Cancer Res. 65: 3509.

Gronostajski R.M. 2000. Roles of the NF1/CTF gene family in transcription and development. Gene 249: 31.

Hammond S.M. 2006. MicroRNAs as oncogenes. Curr. Opin. Genet. Dev. 16: 4.

John B., Enright A.J., Aravin A., Tuschl T., Sander C., and Marks D.S. 2004. Human microRNA targets. PLoS Biol. 2: e363.

Khanna-Gupta A., Zibello T., Sun H., Lekstrom-Himes J., and Berliner N. 2001. C/EBP epsilon mediates myeloid differentiation and is regulated by the CCAAT displacement protein (CDP/cut). Proc. Natl. Acad. Sci. 98: 8000.

Kasashima K., Nakamura Y., and Kozu T. 2004. Altered expres- 
sion profiles of microRNAs during TPA-induced differentiation of HL-60 cells. Biochem. Biophys. Res. Commun. 322: 403.

Kim V.N. 2005. MicroRNA biogenesis: Coordinated cropping and dicing. Nat. Rev. Mol. Cell Biol. 6: 376.

Lee R.C. and Ambros V. 2001. An extensive class of small RNAs in Caenorhabditis elegans. Science 294: 862.

Lee R.C., Feinbaum R.L., and Ambros V. 1993. The C. elegans heterochronic gene lin-4 encodes small RNAs with antisense complementarity to lin-14. Cell 75: 843 .

Lewis B.P., Burge C.B., and Bartel D.P. 2005. Conserved seed pairing, often flanked by adenosines, indicates that thousands of human genes are microRNA targets. Cell 120: 15 .

Lu J., Getz G., Miska E.A., Alvarez-Saavedra E., Lamb J., Peck D., Sweet-Cordero A., Ebert B.L., Mak R.H., Ferrando A.A., et al. 2005. MicroRNA expression profiles classify human cancers. Nature 435: 834.

McKnight S.L. 2001. McBindall-A better name for CCAAT/enhancer binding proteins? Cell 107: 259.

Meisterernst M., Gander I., Rogge L., and Winnacker E.L. 1988. A quantitative analysis of nuclear factor I/DNA interactions. Nucleic Acids Res. 16: 4419.

Metzler M., Wilda M., Busch K., Viehmann S., and Borkhardt A. 2004. High expression of precursor microRNA-155/BIC RNA in children with Burkitt lymphoma. Genes Chromosomes Cancer 39: 167.

Nakajima H. and Ihle J.N. 2001. Granulocyte colonystimulating factor regulates myeloid differentiation through CCAAT/enhancer-binding protein epsilon. Blood 98: 897.

Nerlov C., McNagny K.M., Doderlein G., Kowenz-Leutz E., and Graf T. 1998. Distinct C/EBP functions are required for eosinophil lineage commitment and maturation. Genes Dev.
12: 2413.

Pillai R.S., Bhattacharyya S.N., Artus C.G., Zoller T., Cougot N., Basyuk E., Bertrand E., and Filipowicz W. 2005. Inhibition of translational initiation by Let-7 MicroRNA in human cells. Science 309: 1573.

Radomska H.S., Huettner C.S., Zhang P., Cheng T., Scadden D.T., and Tenen D.G. 1998. CCAAT/enhancer binding protein alpha is a regulatory switch sufficient for induction of granulocytic development from bipotential myeloid progenitors. Mol. Cell. Biol. 18: 4301.

Reddy V.A., Iwama A., Iotzova G., Schulz M., Elsasser A., Vangala R.K., Tenen D.G., Hiddemann W., and Behre G. 2002. Granulocyte inducer C/EBPalpha inactivates the myeloid master regulator PU.1: Possible role in lineage commitment decisions. Blood 100: 483.

Santoro C., Mermod N., Andrews P.C., and Tjian R. 1988. A family of human CCAAT-box-binding proteins active in transcription and DNA replication: Cloning and expression of multiple cDNAs. Nature 334: 218.

Sempere L.F., Freemantle S., Pitha-Rowe I., Moss E., Dmitrovsky E., and Ambros V. 2004. Expression profiling of mammalian microRNAs uncovers a subset of brainexpressed microRNAs with possible roles in murine and human neuronal differentiation. Genome Biol. 5: R13.

Tenen D.G. 2001. Abnormalities of the CEBP alpha transcription factor: A major target in acute myeloid leukemia. Leukemia 4: 688.

Wang X., Scott E., Sawyers C.L., and Friedman A.D. 1999. $\mathrm{C} /$ EBPalpha bypasses granulocyte colony-stimulating factor signals to rapidly induce PU.1 gene expression, stimulate granulocytic differentiation, and limit proliferation in $32 \mathrm{D} \mathrm{cl} 3$ myeloblasts. Blood 94: 560 . 


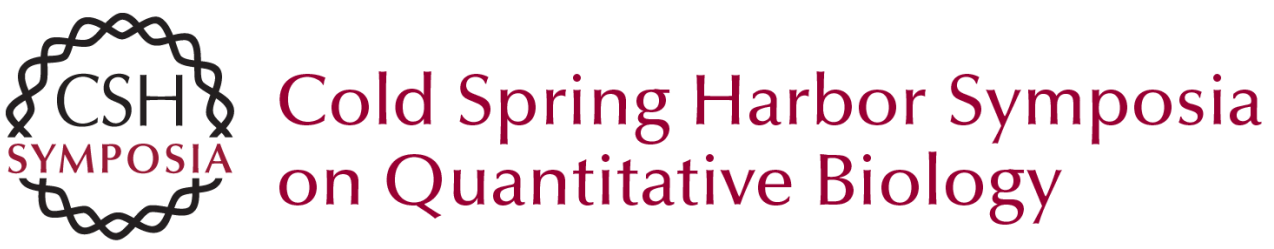

\section{MicroRNAs and Hematopoietic Differentiation}

A. FATICA, A. ROSA, F. FAZI, et al.

Cold Spring Harb Symp Quant Biol 2006 71: 205-210

Access the most recent version at doi:10.1101/sqb.2006.71.014

References This article cites 35 articles, 15 of which can be accessed free at: http://symposium.cshlp.org/content/71/205.full.html\#ref-list-1

\section{License}

Email Alerting Receive free email alerts when new articles cite this article - sign up in Service the box at the top right corner of the article or click here. 